Peter T.-L. Choi MD FRCPC, ${ }^{\star} \ddagger$ Alejandro R. Jadad MD DPHIL FRCPC* $† \ddagger$

\title{
Systematic reviews in anesthesia: should we embrace them or shoot the messenger?
}

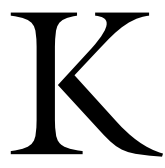

EEPINGup with the anesthesia literature is a daunting task. The body of knowledge is expanding exponentially and most anesthesiologists have limited access to information technology in their working environments, especially in the operating room setting. There are few sources of information and technology that could meet the urgency required by many anesthetic decisions.

Ideally, anesthesiologists should have available strategies that will provide current, valid, and relevant information to guide them in their clinical, research, and administrative decisions, at the right time, in the right place, in the right format and at the right speed. At the very least, these strategies are likely to require unbiased syntheses of the current best information, state-of-the-art information technology, initiatives to optimize the skills with which people use technology and health information, and changes in the environments where anesthesiologists make their decisions to ensure that there is optimal interaction between the best information available and the values, preferences, and circumstances or context in which the decisions are made.

During the past two decades, there has been considerable progress in the way in which research is synthesized. This progress has led to what is called a systematic review. Systematic reviews are reviews that follow a scientific process to reduce bias and increase precision in the collection, appraisal, and interpretation of relevant studies. ${ }^{1}$ For areas where abundant information exists, systematic reviews provide readers with an efficient strategy to deal with overload.

Ideally, a systematic review clearly outlines a focused question, a detailed search strategy, inclusion criteria for its selected studies, an assessment of the quality of the studies upon which the conclusions will be based, and the methods with which the data are amalgamated to produce the conclusions. ${ }^{2}$ Such a review would provide a reader sufficient information with which to evaluate the validity of the conclusions or replicate the review process. As a continuing medical education tool, the systematic review presents the reader with a succinct distillation of a specific body of knowledge. In addition, a systematic review can identify when sufficient research has been conducted to determine the benefit or harm of an intervention much earlier than traditional narrative reviews ${ }^{3}$ and can highlight areas where research must be continued, initiated, or even stopped.

Systematic reviews of postoperative nausea and vom iting (PONV): research vomitus or prevention of research ad nauseum?

PONV is one area in anesthesia where the systematic review has been used extensively to summarize information. The potential benefits are clear. If a person was to find randomized clinical trials (RCTs) on PONV, she or he would find 346 reports of individual RCTs in the MEDLINE database just for the 1990s with the yearly number increasing from 11 reports in 1990 to 52 reports in 1999. Even at half an hour per article, such a person would need to devote over a week of uninterrupted time to read all the published clinical trials of just the last decade! In contrast, 22 systematic reviews (including the one in this issue) have been published summarizing the evidence behind some of the risk factors and interventions for PONV (Table). ${ }^{4-25}$ Specific patient populations, nonpharmacological interventions, and drugs have been reviewed by this process.

What have we learnt from these reviews? Several reviews inform us that omitting nitrous oxide from our anesthetic will reduce the incidence of PONV, ${ }^{6,8}$ but the incidence of intraoperative awareness will

From the Departments of Anaesthesia* and Clinical Epidemiology \& Biostatistics, $†$ McMaster University; and the Program in Evidencebased Care, $\ddagger$ Cancer Care Ontario, Hamilton, Ontario, Canada.

Address correspondence to: Dr. Alejandro R. Jadad, Department of Clinical Epidemiology \& Biostatistics, McMaster University, HSC3H7, 1200 Main Street West, Hamilton, Ontario, L8N $3 Z 5$ Canada. Phone: 905-525-9140, ext 22197; Fax: 905-546-0401; Email: jadada@fhs.mcmaster.ca

CAN J ANESTH 2000/47: $6 /$ pp 486-493 
TABLE List of published systematic reviews on postoperative nausea and vomiting

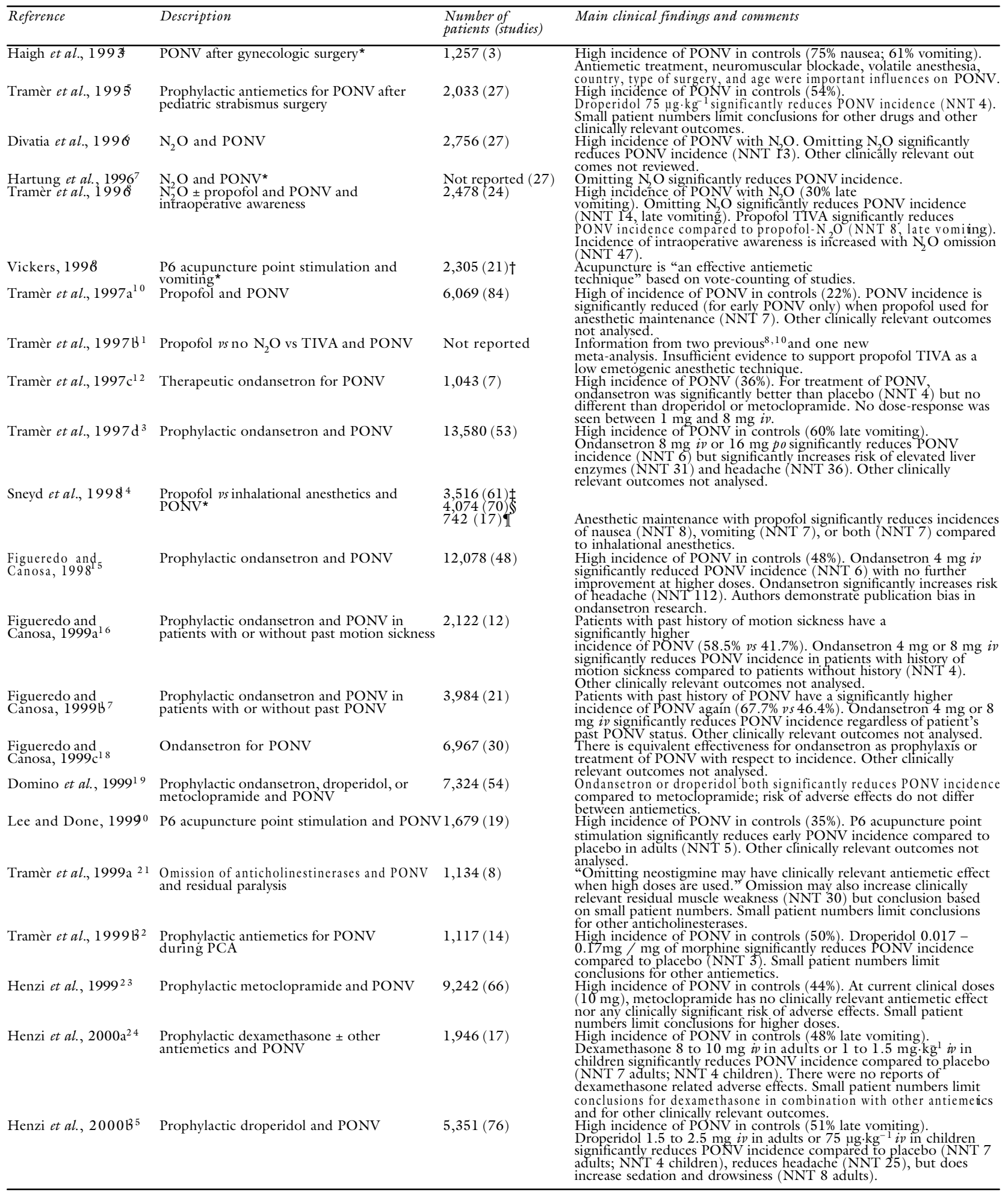

Abbreviations: $i v$ - intravenous; NNT - number needed to treat (number of patients receiving intervention before one episode of the outcome of interest is observed); $\mathrm{N}_{2} \mathrm{O}$ - nitrous oxide; PCA - patient controlled analgesia; po - per os (oral); PONV - postoperative nausea and vomiting; TIVA - total intravenous

${ }^{*}$ Major or extensive flaws in methodology or reporting limit the validity of this review.

Numbers are for PONV trials.

Numbers are for nausea as the primary outcome.

Numbers are for vomiting as the primary outcome.
Numbers are for nausea and vomiting as the primary outcome. 
increase. ${ }^{8}$ However, the overall reduction in PONV will be higher than the risk of awareness. ${ }^{8}$ Similarly, systematic reviews highlight that the limited evidence available suggests that there is no difference in therapeutic effect between ondansetron, droperidol, or metoclopramide and shows no dose-response for therapeutic ondansetron. ${ }^{12}$ Additional findings from systematic reviews of PONV are highlighted in the Table.

In this issue, Henzi et al. ${ }^{25}$ review the evidence from all RCTs comparing droperidol with placebo or no treatment in surgical patients as a prophylaxis or therapy for PONV (classified as early and late). Adverse effects of droperidol were also assessed. The conclusions were drawn from a total of 5,351 patients from 76 trials.

What clinical lessons can we learn from this systematic review? First, there is a high incidence of PONV in the surgical population. The incidence of PONV within the first six (early) and the first $24 \mathrm{hr}$ (late) after surgery averaged $34 \%$ and $51 \%$ respectively! The reviewers found that droperidol was efficacious in treating nausea and vomiting. At a dose of $2.5 \mathrm{mg}$ in adult patients, five patients needed to receive prophylaxis to prevent one episode of nausea and seven needed to receive prophylaxis to prevent one episode of early or late vomiting. In children, $75 \mu \mathrm{g} \cdot \mathrm{kg}^{-1}$ of droperidol given to four patients would prevent one episode of early or late vomiting. The incidence of extrapyramidal symptoms is approximately 1 in 90 for children and 1 in 400 for all patients. Incidence of sedation and drowsiness was approximately 1 in 8 for adults. Dose-responsiveness was observed for prevention of vomiting and for sedation and drowsiness.

For those interested in methodology, some additional lessons can be drawn from this systematic review. The review highlights the difficulty in assessing the natural history of PONV and the effectiveness of antiemetics using information from current research. Although there is interesting data on the incidence of PONV, there is little on the duration of PONV following prophylactic droperidol compared to placebo. Data on patient satisfaction, duration of recovery room stay, unplanned hospital admissions, or costs were not reported. The inability to answer these questions is not a weakness of the systematic review method. The problem lies in the choice of clinical outcomes used in the trials reviewed (and many PONV trials in general). As others have pointed out, ${ }^{26,27} \mathrm{clin}$ ically relevant outcomes rather than surrogate measures are needed in PONV trials.
Interpreting systematic reviews: shoot the messenger or shoot the researcher?

How should readers react to information provided by systematic reviews? Ideally, readers should react in a positive way, realizing the limitations of knowledge available. But realistically, we do not know if clinicians are receptive to messages from systematic reviews that repeatedly highlight uncertainty and increased potential for bias in research. In fact, editorials in the anesthetic literature have criticized systematic reviews. ${ }^{26,27}$ Why? Are they attacking the tool (on methodological or clinical grounds) or are they reacting to the distress generated by uncertainty and the realization of the limits of our knowledge? Is this shooting the messenger?

Admittedly, systematic reviews are difficult to read and interpret compared to narrative reviews. Often, they leave the reader without a definite answer, confused, perhaps frustrated, and suspicious of the value of the entire evidence-based process. These are understandable and important concerns but the challenges are not insurmountable. The solution to these issues is not to scrap systematic reviews. By making limitations of research explicit, systematic reviews should enable the decision-makers to become aware of the state of the "best evidence" available, helping them acknowledge that their decisions are largely based on clinical data and experience.

Instead of ignoring or even suppressing systematic reviews, we should embrace them and use them as guides to improve the evidence upon which they attempt to draw inferences. In addition, we should put more emphasis on efforts to increase their readerfriendliness and quality of reporting, ${ }^{28}$ and promote strategies to increase the reader's skills in interpreting and applying information from this type of literature. ${ }^{2,29}$

Finally, we need to acknowledge that systematic reviews and traditional narrative reviews are complementary means of acquiring knowledge. A good narrative review is like an impressionistic landscape: the reader is informed of the broad scope and issues of a particular topic but details may be fuzzy or slanted toward a specific point of view. In contrast, a good systematic review is like a classical master drawing: the reader is provided structured, detailed information of a specific issue of a topic but sometimes the relationship of the issue to its whole may be unclear. Each has a role in our continuing education.

The design and execution of systematic reviews require special skills, and considerably more time and resources than do their narrative counterparts. This is why it is so important to avoid duplication of work by encouraging collaboration among individuals around the world interested in reviewing the same topics. 
Achieving this successfully and efficiently may soon stop being science fiction. An international group of people interested in systematic reviews in anesthesia met in Amsterdam in 1999. This collaborative initiative known as the Cochrane Anaesthesia Review Group (http://www.cochrane-anaesthesia.suite.dk) operates within the Cochrane Collaboration (http://hiru.mcmaster.ca/cochrane) and is committed to meeting the challenges in anesthesia by preparing, maintaining, and facilitating the accessibility of up-todate reviews of health care interventions related to anesthesia. Instead of getting sick over the thought of the massive and growing body of anesthetic knowledge, we hope that this initiative will motivate anesthesiologists worldwide to join efforts and pursue the best knowledge for the practice of evidence-based anesthesia.

Acknowledgments

Dr. Jadad was funded by the Program in Evidencebased Care, Cancer Care Ontario, Toronto; and a National Health Scholar Award from Health Canada, Ottawa.

\section{Les révisions systéma- tiques en anesthésie : les adopter ou les éliminer ?}

Se tenir à jour en anesthésie, c'est un travail impressionnant. L'ensemble des connaissances s'accroît de façon exponentielle et la plupart des anesthésiologistes n'ont qu'un accès limité à la technologie de l'information dans leur environnement de travail, surtout à la salle d'opération. Il existe peu de sources d'information et de technologie capables de répondre à l'urgence exigée par de nombreuses décisions anesthésiques.

Idéalement, les anesthésiologistes devraient bénéficier de mesures accessibles qui fournissent l'information courante, valide et pertinente pouvant les guider dans leurs décisions en clinique, en recherche et en administration, au bon moment, au bon endroit, selon la présentation et la vitesse appropriées. À tout le moins, ces stratégies nécessitent des synthèses non biaisées de la meilleure information actuelle, de la technologie de pointe en information, des initiatives pour optimaliser la qualité d'utilisation de la technologie et de l'information sur la santé et des changements dans l'environnement où les anesthésiologistes pren- nent leurs décisions. Tout cela dans le but de permettre l'interaction maximale entre les meilleures informations disponibles et les valeurs, les préférences et les circonstances, ou le contexte, dans lequel les décisions sont prises.

Pendant les deux dernières décennies, il y a eu des progrès considérables dans la manière de faire la synthèse des recherches. Ce progrès a conduit à ce qu'on appelle une révision systématique. Les révisions systématiques suivent un processus scientifique qui réduit le biais et accroit la précision dans la cueillette, l'appréciation et l'interprétation des études retenues. ${ }^{1}$ Dans le cas des domaines qui foisonnent d'informations, les révisions systématiques fournissent aux lecteurs une stratégie efficace pour faire face à la surabondance.

Au mieux, une révision systématique indique une question centrale, une stratégie de recherche détaillée, un critère d'inclusion pour les études choisies, une évaluation de la qualité des études sur lesquelles les conclusions seront basées et les méthodes avec lesquelles les données ont été réunies pour produire les conclusions. ${ }^{2}$ Une révision de ce type fournit des informations suffisantes pour permettre d'évaluer la validité des conclusions ou de reproduire le processus de révision. Comme outil de formation médicale continue, la révision systématique présente au lecteur le condensé d'un aspect spécifique du savoir. En outre, une révision systématique peut dire plus rapidement qu'une révision classique de textes si une recherche suffisante a été menée dans le but de déterminer l'avantage ou l'inconvénient d'une intervention ${ }^{3}$ et peut souligner les domaines dans lesquels la recherche devra se poursuivre, s'amorcer ou même s'arrêter.

Les révisions systématiques des nausées et des vom issements postopératoires (NVPO) : rejet de la recherche ou mesures préventives de recherche à donner la nausée ?

Les NVPO représentent un champ d'anesthésie où la révision systématique a été beaucoup utilisée pour résumer l'information. Les avantages potentiels sont clairs. Si une personne cherche des essais cliniques randomisés (ECR) sur les NVPO, elle trouvera 346 articles sur des ECR individuels dans la base de données de MEDLINE, seulement pour les années 1990, dont un nombre annuel croissant allant de 11 articles en 1990 jusqu'à 52 en 1999. Même en ne passant qu'une demi-heure par article, cette personne devra prendre plus d'une semaine de travail ininterrompu pour lire tous les essais cliniques publiés dans la dernière décennie uniquement. Par contraste, 22 révisions systématiques (incluant la révision du présent numéro) publiés résument les arguments présentés pour certains fac- 
TABLE Liste des révisions systématiques publiées sur les nausées et les vomissements postopératoires

\begin{tabular}{|c|c|c|c|}
\hline Référence & $\begin{array}{l}\text { Description } \\
\text { patients (études) }\end{array}$ & Nombre & $\begin{array}{l}\text { Constatations cliniques principales et } \\
\text { commentaires }\end{array}$ \\
\hline Haigh et coll., $1993^{4}$ & NVPO en gynécologie* & $1257(3)$ & $\begin{array}{l}\text { Forte incidence de NVPO chez les témoins ( } 75 \% \text { nausées; } 61 \% \\
\text { vomissements). Le traitement antiémétique, le bloc neuromus- } \\
\text { culaire, l'anesthésie volatile, le pays, le type d'opération et l'âge } \\
\text { ont d'importantes influences sur les NVPO. }\end{array}$ \\
\hline Tramèr et coll., 1995 & $\begin{array}{l}\text { Antiémétiques prophylactiques pour les NVPO } \\
\text { après correction d'un strabisme pédiatrique }\end{array}$ & $2033(27)$ & 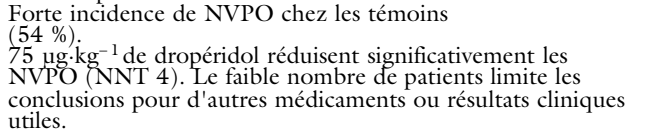 \\
\hline Divatia et coll., 1998 & $\mathrm{~N}_{2} \mathrm{O}$ et $\mathrm{NVPO}$ & $2756(27)$ & $\begin{array}{l}\text { Forte incidence de NVPO avec } \mathrm{N}_{2} \mathrm{O} \text {. Sans } \mathrm{N}_{2} \mathrm{O} \text {, réduction } \\
\text { significative des NVPO (NNT } 13)^{2} \text {. D'autres résultats cliniques } \\
\text { applicables non révisés. }\end{array}$ \\
\hline Hartung et coll., 1996 & $\mathrm{~N}_{2} \mathrm{O}$ et NVPO* & Non cité (27) & Sans $\mathrm{N}_{2} \mathrm{O}$, réduction significative des NVPO. \\
\hline Tramèr et coll., 1998 & $\begin{array}{l}\mathrm{N}_{2}^{2} \mathrm{O} \pm \text { propofol et NVPO et } \\
\text { conscience opératoire }\end{array}$ & $2478(24)$ & $\begin{array}{l}\text { Forte incidence de NVPO avec } \mathrm{N}_{2} \mathrm{O}(30 \% \\
\text { vomissements tardifs). Sans } \mathrm{N}_{2} \mathrm{O} \text {, réduction significative des } \\
\text { NVPO (NNT 14, vomissements tardifs). L'AEIV au propofol } \\
\text { réduit significativement les NVPO comparé au propofol-N, } \mathrm{O} \\
\text { (NNT 8, vomissements tardifs). Sans } \mathrm{N}_{2} \mathrm{O} \text {, augmentation de } \\
\text { conscience peropératoire (NNT } 47 \text { ). }\end{array}$ \\
\hline Vickers, 1998 & $\begin{array}{l}\text { Stimulation d'acupuncture P6 et } \\
\text { vomissements }\end{array}$ & $2305(21) \dagger$ & $\begin{array}{l}\text { L'acupuncture est une «technique } \\
\text { antiémétique efficace» basée sur le dépouillement des votes des } \\
\text { études. }\end{array}$ \\
\hline Tramèr et coll., $1997 \mathrm{a}^{10}$ & Propofol et NVPO & $6069(84)$ & $\begin{array}{l}\text { Forte incidence de NVPO chez les témoins ( } 22 \%) \text {. L'incidence } \\
\text { des NVPO est significativement réduite (pour les NVPO } \\
\text { précoces seuls) avec du propofol pour maintenir l'anesthésie } \\
\text { (NNT 7) D'autres résultats cliniques applicables non analysés. }\end{array}$ \\
\hline Tramèr et coll., $1997 \mathrm{~b}^{1}$ & Propofol vs sans $\mathrm{N}_{2} \mathrm{O}$ vs AEIV et NVPO & Non cité & Informations de deux méta-analyses anterieures ${ }^{8,10}$ et une \\
\hline Tramèr et coll., $1997 \mathrm{c}^{12}$ & Ondansétron thérapeutique pour les NVPO & $1043(7)$ & $\begin{array}{l}\text { Preuve insuffisante pour accepter l'AEIV au propofol comme } \\
\text { une technique faiblement émétique. } \\
\text { Forte incidence de NVPO ( } 36 \%) \text {. Pour traiter les NVPO, } \\
\text { l'ondansétron a été significativement meilleur que le placebo } \\
\text { (NNT 4), mais non différent du dropéridol ou du } \\
\text { métoclopramide. Pas de réponse liée à la dose avec de } 1 \mathrm{mg} \text { à } \\
8 \mathrm{mg} \text { iv. }\end{array}$ \\
\hline Tramèr et coll., $1997 \mathrm{~d}^{3}$ & Ondansétron prophylactique et NVPO & $13580(53)$ & $\begin{array}{l}\text { Forte incidence de NVPO chez les témoins ( } 60 \% \text { de } \\
\text { vomissements tardifs). } 8 \mathrm{mg} \text { iv d'ondansétron ou } 16 \text { mg po } \\
\text { réduisent significativement les NVPO (NNT 6), mais } \\
\text { augmentent significativement le risque d'enzymes hépatiques } \\
\text { élevés (NNT 31) et de céphalées (NNT 36). D'autres résultats } \\
\text { cliniques applicables non analysés. }\end{array}$ \\
\hline Sneyd et coll., $1998^{4}$ & $\begin{array}{l}\text { Propofol vs anesthésiques d'inhalation et } \\
\text { NVPO* }\end{array}$ & $\begin{array}{l}3516(61) \ddagger \\
4074(70) \$ 5 \\
742(17)\end{array}$ & Le maintien anesthésique avec propofol réduit significativement \\
\hline Figueredo et Canosa, $1998^{15}$ & Ondansétron prophylactique et NVPO & $12078(48)$ & $\begin{array}{l}\text { les nausées (NNT 8), les vomissements (NNT 7) ou les deux } \\
\text { (NNT 7) comparé aux anesthésiques d'inhalation. } \\
\text { Forte incidence de NVPO chez les témoins ( } 48 \text { \%). } 4 \text { mg iv } \\
\text { d'ondansétron réduisent significativement les NVPO (NNT 6) } \\
\text { sans autre amélioration pour des doses plus élevées. } \\
\text { L'ondansétron augmente significativement le risque de } \\
\text { céphalées (NNT 112). Les auteurs ont démontré des } \\
\text { biais dans les recherches sur l'ondansétron. }\end{array}$ \\
\hline (1) & $\begin{array}{l}\text { Ondansétron prophylactique et NVPO } \\
\text { avec ou sans NVPO antérieurs }\end{array}$ & $2122(12)$ & $\begin{array}{l}\text { Patients avec antécédents de mal des transports ont une } \\
\text { incidence significativement plus élevée de NVPO (58,5\% vs } \\
41,7 \%) .4 \mathrm{mg} \text { ou } 8 \mathrm{mg} \text { iv d'ondansétron réduisent } \\
\text { significativement les NVPO chez les patients avec histoire de } \\
\text { mal des transports comparés aux patients sans antécédents } \\
\text { (NNT } 4 \text { ). } \\
\text { D'autres résultats cliniques applicables non } \\
\text { analysés. }\end{array}$ \\
\hline Figueredo et Canosa, 1999 b & $\begin{array}{l}\text { Ondansétron prophylactique et NVPO chez } \\
\text { ou sans NVPO antérieurs }\end{array}$ & $3984(21)$ & $\begin{array}{l}\text { Les patients avec antécédents de NVPO ont } \\
\text { significativement plus de } \\
\text { NVPO }(67,7 \% \text { vs } 46,4 \%) .4 \mathrm{mg} \text { ou } 8 \mathrm{mg} \text { iv d'ondansétron } \\
\text { réduisent significativement les NVPO peu importe la situation } \\
\text { antérieure des NVPO. D'autres résultats cliniques applicables } \\
\text { non analysés. }\end{array}$ \\
\hline Figueredo et Canosa, $1999 \mathrm{c}^{18}$ & Ondansetron pour NVPO & $6967(30)$ & $\begin{array}{l}\text { Efficacité équivalente de l'ondansétron comme prophylaxie ou } \\
\text { traitement des NVPO. D'autres résultats cliniques } \\
\text { applicables non analysés. }\end{array}$ \\
\hline Domino et coll., $1999^{9}$ & $\begin{array}{l}\text { Ondansétron ou dropéridol prophylactiques } \\
\text { métoclopramide et NVPO }\end{array}$ & $7324(54)$ & $\begin{array}{l}\text { Ondansétron ou dropéridol réduisent } \\
\text { significativement les NVPO comparé au métoclopramide; } \\
\text { mémes risques d'effets secondaires entre les antiemétiques. }\end{array}$ \\
\hline Lee et Done, $1999^{\circ}$ & Point de stimulation d'acupuncture P6 et NVPO & $1679(19)$ & $\begin{array}{l}\text { Forte incidence de NVPO chez les témoins (35\%). Stimulation } \\
\text { d'acupuncture au point P6 réduit significativement les NVPO } \\
\text { précoces comparée au placebo chez les adultes (NNT } 5 \text { ). } \\
\text { D'autres résultats cliniques applicables non analysés. }\end{array}$ \\
\hline Tramèr et coll., $1999 \mathrm{a}^{21}$ & $\begin{array}{l}\text { Omission d'anticholinestérases et NVPO } \\
\text { et paralysie résiduelle }\end{array}$ & $1134(8)$ & $\begin{array}{l}\text { «L'omission de néostigmine peut avoir un } \\
\text { effet antiémétique clinique significatif avec de fortes } \\
\text { doses.» L'omission peut aussi augmenter la faiblesse musculaire } \\
\text { résiduelle de façon significative (NNT 30), mais les } \\
\text { conclusions sont basees sur un faible nombre de patients. Le } \\
\text { faible nombre de patients limite les conclusions pour d'autres } \\
\text { anticholinestérases. }\end{array}$ \\
\hline Tramèr et coll., 1999 $\mathrm{B}^{2}$ & $\begin{array}{l}\text { Antiémétiques prophylactiques pour les NVPO } \\
\text { pendant l'ACP }\end{array}$ & $1117(14)$ & $\begin{array}{l}\text { Forte incidence de NVPO chez les témoins pendant l'ACP } \\
(50 \%) \text {. Du dropéridol à } 0,017-0,17 \mathrm{mg} / \mathrm{mg} \text { de morphine } \\
\text { réduit significativement les NVPO comparé au placebo } \\
\text { (NNT 3). Le faible nombre de patients limite les conclusions } \\
\text { pour d'autres antiémétiques. }\end{array}$ \\
\hline Henzi et coll., $1999^{3}$ & $\begin{array}{l}\text { Le métoclopramide prophylactique } \\
\text { et NVPO }\end{array}$ & $9242(66)$ & $\begin{array}{l}\text { Forte incidence de NVPO chez les témoins (44\%). Pour des } \\
\text { doses habituelles (10 mg), le métoclopramide n'a pas d'effet } \\
\text { antiémétique clinique significatif ni de risque significatif } \\
\text { d'effet secondaire. Le faible nombre de patients limite les } \\
\text { conclusions pour des doses plus élevées. }\end{array}$ \\
\hline
\end{tabular}


TABLE continue

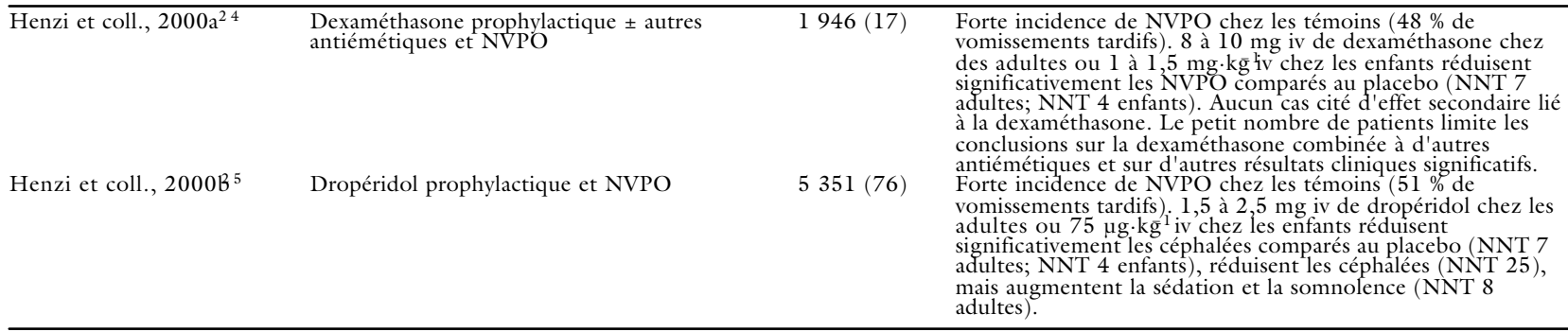

Abréviations: $i v$ - intraveineux; NNT - nombre nécessaire à traiter (nombre de patients qui reçoivent une intervention avant qu'un épisode d'un événement d'intérêt ne soit observé); $\mathrm{NO}_{2}$ - protoxyde d'azote; ACP - analgésie contrôlée par le patient; po - per os (oral); NVPO - nausées et vomissements postopératoires; AEIV - anesthésie essentiellement intraveineuse.

* Des inexactitudes importantes ou nombreuses de méthodologie ou de présentation limitent la validité de cette révision.

$\dagger$ Les nombres indiquent des essais sur les NVPO.

‡ Les nombres indiquent les nausées comme principale observation.

$\$$ Les nombres indiquent les vomissements comme principale observation.

If Les nombres indiquent les nausées et les vomissements comme principale observation.

teurs de risque et interventions dans le cas des NVPO (Table). ${ }^{4-25}$ Des populations spécifiques de patients, des interventions non pharmacologiques et des médicaments ont été passés en revu de cette manière.

Qu'avons-nous appris de ces révisions ? Certaines révisions nous disent que de retirer le protoxyde d'azote de nos anesthésiques pourra réduire l'incidence des NVPO $,{ }^{6}, 8$ mais que l'incidence de conscience peropératoire va augmenter. ${ }^{8}$ Cependant, la réduction totale de NVPO sera plus forte que le risque de conscience. ${ }^{8}$ De même, les révisions systématiques soulignent que le nombre limité de preuves présentées suggère qu'il n'y a pas de différence d'effet thérapeutique entre l'ondansétron, le dropéridol ou le métoclopramide. ${ }^{12}$ Des constatations supplémentaires provenant d'autres révisions systématiques des NVPO sont mises en relief dans la Table.

Dans le présent numéro, Henzi et coll. ${ }^{25}$ passent en revue les observations de tous les ECR qui comparent le dropéridol, comme mesure prophylactique ou thérapeutique des NVPO (classifiés comme précoces ou tardifs), avec un placebo ou l'absence de traitement chez des opérés. Les effets indésirables du dropéridol sont aussi évalués. Les conclusions proviennent d'un total de 76 essais auprès de 5351 patients.

Quelles leçons cliniques pouvons-nous tirer de cette révision systématique ? D'abord, il y a une forte incidence de NVPO parmi la population chirurgicale. L'incidence de NVPO pendant les six premières heures (précoces) et les 24 premières heures (tardifs) postopératoires est en moyenne de $34 \%$ et de $51 \%$, respectivement ! Les réviseurs ont trouvé que le dropéridol était efficace contre les nausées et les vomissements. Selon une dose de $2,5 \mathrm{mg}$ chez des adultes, cinq patients ont eu besoin de prophylaxie pour prévenir un épisode de nausées et sept pour prévenir un épisode de vomissements précoces ou tardifs. Chez les enfants, $75 \mu \mathrm{g} \cdot \mathrm{kg}^{-1}$ de dropéridol administrés à quatre patients ont empêché des vomissements précoces ou tardifs. L'incidence de symptômes extrapyramidaux est d'environ 1 sur 90 chez les enfants et de 1 sur 400 pour tous les patients. L'incidence de sédation et de somnolence était d'environ 1 adulte sur 8 . La réactivité posologique a été observée dans les cas de prévention des vomissements, et de sédation et de somnolence.

Pour ceux que la méthodologie intéresse, des leçons supplémentaires peuvent être tirées de cette révision systématique. La révision souligne la difficulté d'évaluer l'histoire naturelle des NVPO et l'efficacité des antiémétiques en utilisant les informations contenues dans les recherches courantes. Même s'il y a des données intéressantes sur l'incidence des NVPO, il en existe peu sur leur durée après l'usage de dropéridol prophylactique comparé au placebo. Les données sur la satisfaction du patient, la durée du séjour en salle de réveil, le nombre d'admissions hospitalières non planifiées ou le coût ne sont pas mentionnés. L'incapacité de répondre à ces questions n'est pas une faiblesse de la méthode de révision systématique. Le problème vient du choix des résultats cliniques utilisés dans les essais passés en revue (et de nombreux essais sur les NVPO en général). Comme d'autres l'ont fait remarquer, ${ }^{26,27}$ dans les essais sur les NVPO, on a besoin de résultats cliniques utiles plutôt que des mesures porteuses.

L'interprétation des révisions systématiques : éliminer l'outil ou le chercheur ?

Comment doit-on réagir aux informations fournies par les révisions systématiques ? Dans le meilleur des cas, les lecteurs devraient avoir une réaction positive tout en se 
rendant compte des limites des connaissances existantes. Mais de façon réaliste, nous ne savons pas si les cliniciens sont réceptifs aux messages des révisions systématiques qui, à répétition, relèvent l'incertitude et le potentiel accru de biais en recherche. De fait, les éditoriaux de la documentation anesthésique ont attaqué les révisions systématiques. ${ }^{26,27}$ Pourquoi ? S'attaquent-ils à l'outil (sur des bases méthodologiques ou cliniques) ou réagissent-ils à la détresse générée par l'incertitude et la prise de conscience des limites du savoir ? Est-ce tirer sur le messager?

Il faut l'admettre, les révisions systématiques sont difficiles à lire et à interpréter en comparaison des révisions textuelles. Souvent, elles laissent le lecteur sans réponse définitive, confus, peut-être insatisfait et méfiant quant à la valeur de toute cette démarche basée sur la preuve. Ce sont des préoccupations compréhensibles et importantes, mais les défis ne sont pas insurmontables. La solution n'est pas d'abandonner les révisions systématiques. En rendant les limites de la recherche explicites, les révisions systématiques devraient aider les décideurs à prendre conscience de l'état de la «meilleure preuve» existante, ce qui peut les aider à reconnaître que leurs décisions sont grandement basées sur des données et de l'expérience cliniques.

Au lieu de ne pas respecter ou même de supprimer les révisions systématiques, nous devrions les adopter et les utiliser comme des guides pour améliorer la preuve à partir de laquelle on tente de tirer des déductions. Qui plus est, nous devrions accentuer nos efforts pour améliorer leur convivialité et la qualité de leur présentation $^{28}$ et promouvoir des plans d'action pour accroître les habiletés des lecteurs à interpréter et appliquer l'information obtenue de ce genre de documentation. ${ }^{2,29}$

Enfin, nous devons reconnaître que les révisions systématiques et les révisions textuelles traditionnelles sont des moyens complémentaires d'acquérir des connaissances. Une bonne révision textuelle est comme un paysage impressionniste : le lecteur est informé de la vaste étendue et des questions relatives à un sujet particulier, mais les détails peuvent être vagues ou déformés en faveur d'un point de vue spécifique. Par ailleurs, une bonne révision systématique est comme un tableau de maître classique : le lecteur reçoit de l'information structurée, détaillée sur un aspect d'un sujet, mais parfois la relation entre la question et l'ensemble du sujet n'est pas claire. Chaque révision a son rôle dans notre formation continue.

La conception et la réalisation de révisions systématiques exigent des qualités spéciales et beaucoup plus de temps et de ressources que leur contrepartie textuelle. C'est pourquoi il est important d'éviter la répétition du travail en encourageant la collaboration de tous ceux qui, à travers le monde, sont intéressés à réviser les mêmes sujets. La réussite et l'efficacité d'une telle entreprise ne seront bientôt plus de la science fiction. Un groupe international de gens intéressés à la révision systématique en anesthésie s'est réuni à Amsterdam en 1999. Cette initiative de collaboration connue sous le nom de Cochrane Anaesthesia Review Group (http://www.cochraneanaesthesia.suite.dk) fait partie de Cochrane Collaboration (http://hiru.mcmaster.ca/cochrane) qui s'est engagé à relever le défi en anesthésie. Leur travail vise à préparer, maintenir et faciliter l'accessibilité aux révisions actualisées des interventions en soins de santé reliées à l'anesthésie. Au lieu de se décourager devant l'ensemble croissant et imposant des connaissances anesthésiques, nous espérons que cette initiative motivera les anesthésiologistes de partout à collaborer et à rechercher ce qu'il y a de mieux pour la pratique de l'anesthésie basée sur l'évidence scientifique.

\section{Remerciements}

Dr. Jadad a reçu une subvention du Program in Evidence-based Care, Cancer Care Ontario à Toronto et un National Health Scholar Award de Santé Canada à Ottawa.

\section{References}

1 Cook DJ, Sackett DL, Spitzer WO. Methodologic guidelines for systematic reviews of randomized control trials in health care from the Potsdam Consultation on meta-analysis. J Clin Epidemiol 1995; 48: 167-71.

2 Oxman AD, Guyatt GH. Guidelines for reading literature reviews. CMAJ 1988; 138: 697-703.

3 Antman EM, Lau J, Kupelnick B, Mosteller F, Chalmers TC. A comparison of results of meta-analyses of randomized controlled trials and recommendations of clinical experts. Treatments for myocardial infarction. JAMA 1992; 268: 240-8.

4 Haigh CG, Kaplan LA, Durbam JM, Dupeyron JP, Harmer M, Kenny GN. Nausea and vomiting after gynaecological surgery: a meta-analysis of factors affecting their incidence. Br J Anaesth 1993; 71: 517-22.

5 Tramèr M, Moore A, McQuay $H$ Prevention of vomiting after paediatric strabismus surgery: a systematic review using the numbers-needed-to-treat method. $\mathrm{Br}$ J Anaesth 1995; 75: 556-61.

6 Divatia JV, Vaidya JS, Badwe RA, Hawaldar RW. Omission of nitrous oxide during anesthesia reduces the incidence of postoperative nausea and vomiting. A meta-analysis. Anesthesiology 1996; 85: 1055-62.

7 Hartung J. Twenty-four of twenty-seven studies show a greater incidence of emesis associated with nitrous oxide than with alternative anesthetics. Anesth Analg 1996; 83: 114-6. 
8 Tramèr M, Moore A, McQuay $H$. Omitting nitrous oxide in general anaesthesia: meta-analysis of intra- operative awareness and postoperative emesis in randomized controlled trials. Br J Anaesth 1996; 76: 186-93.

9 Vickers AJ. Can acupuncture have specific effects on health? A systematic review of acupuncture antiemesis trials. J R Soc Med 1996; 89: 303-11.

10 Tramèr M, Moore A, McQuay $H$ Propofol anaesthesia and postoperative nausea and vomiting: quantitative systematic review of randomized controlled studies. $\mathrm{Br}$ J Anaesth 1997; 78: 247-55.

11 Tramèr M, Moore A, McQuay H. Meta-analytic comparison of prophylactic antiemetic efficacy for postoperative nausea and vomiting: propofol anesthesia vs omitting nitrous oxide vs total i.v. anaesthesia with propofol. Br J Anaesth 1997; 78: 256-9.

12 Tramèr MR, Moore RA, Reynolds DJ, McQuay HJ. A quantitative systematic review of ondansetron in treatment of established postoperative nausea and vomiting. BMJ 1997; 314: 1088-92.

13 Tramèr MR, Reynolds DJ, Moore RA, McQuay HJ. Efficacy, dose response, and safety of ondansetron in prevention of postoperative nausea and vomiting: a quantitative systematic review of randomized placebocontrolled trials. Anesthesiology 1997; 87: 1277-89.

14 Sneyd JR, Carr A, Byrom WD, Bilski AJ. A meta-analysis of nausea and vomiting following maintenance of anaesthesia with propofol or inhalational agents. Eur J Anaesthesiol 1998; 15: 433-45.

15 Figueredo ED, Canosa LG. Ondansetron in the prophylaxis of postoperative vomiting: a meta-analysis. J Clin Anesth 1998; 10: 211-21.

16 Figueredo E, Canosa L. Prophylactic ondansetron for post-operative emesis: meta-analysis of its effectiveness in patients with and without a previous history of motion sickness. Eur J Anaesthesiol 1999; 16: 556-64.

17 Figueredo E, Canosa L. Prophylactic ondansetron for postoperative emesis. Meta-analysis of its effectiveness in patients with previous history of postoperative nausea and vomiting. Acta Anaesthesiol Scand 1999; 43: 637-44.

18 Figueredo E, Canosa LG. Prevention or treatment of postoperative vomiting using ondansetron? A mathematical assessment. J Clin Anesth 1999; 11: 24-31.

19 Domino KB, Anderson EA, Polissar NL, Posner KL. Comparative efficacy and safety of ondansetron, droperidol, and metoclopramide for preventing postoperative nausea and vomiting: a meta-analysis. Anesth Analg 1999; 88: 1370-9.

20 Lee A, Done $M L$. The use of nonpharmacologic techniques to prevent postoperative nausea and vomiting: a meta-analysis. Anesth Analg 1999; 88: 1362-9.

21 Tramèr MR, Fuchs-Buder T. Omitting antagonism of neuromuscular block: effect of postoperative nausea and vomiting and risk of residual paralysis. A systematic review. Br J Anaesth 1999; 82: 379-86.

22 Tramèr MR, Walder B. Efficacy and adverse effects of prophylactic antiemetics during patient-controlled analgesia therapy: a quantitative systematic review. Anesth Analg 1999; 88: 1354-61.

23 Henzi I, Walder B, Tramèr MR. Metoclopramide in the prevention of postoperative nausea and vomiting: a quantitative systematic review of randomized, placebocontrolled studies. Br J Anaesth 1999; 83: 761-71.

24 Henzi I, Walder B, Tramèr MR. Dexamethasone for the prevention of postoperative nausea and vomiting: a quantitative systematic review. Anesth Analg 2000; 90: 186-94.

25 Henzi I, Sonderegger J, Tramèr MR. Efficacy, doseresponse, and adverse effects of droperidol for prevention of postoperative nausea and vomiting. A quantitative systematic review. Can J Anaesth 2000; 47: (ANDREW, PLEASE PAGE NUMBERS).

26 Fisher DM. The "big little problem" of postoperative nausea and vomiting. Do we know the answer yet? (Editorial) Anesthesiology 1997; 87: 1271-3.

27 White PF, Watcha MF. Has the use of meta-analysis enhanced our understanding of therapies for postoperative nausea and vomiting? (Editorial) Anesth Analg 1999; 88: 1200-2.

28 Moher D, Cook DJ, Eastwood S, Olkin I, Rennie D, Stroup DF. Improving the quality of reports of metaanalyses of randomized controlled trials: the QUOROM statement. Quality of reporting of metaanalyses. Lancet 1999; 354: 1896-900.

29 Oxman AD, Cook DJ, Guyatt GH. Users' guides to the medical literature: VI. How to use an overview. Evidence-Based Medicine Working Groupl JAMA 1994; 272: 1367-71. 